\title{
Thyroid antibodies and risk of preterm delivery: a meta-analysis of prospective cohort studies
}

\author{
Xiaoyan He, Pingping Wang ${ }^{1}$, Zengfang Wang ${ }^{1}$, Xiaoqin $\mathrm{He}^{1}$, Donghua $\mathrm{Xu}^{2}$ and Bin Wang ${ }^{1}$ \\ Department of Clinical Laboratory, People's Hospital of Ganzhou, Ganzhou 341000, People's Republic of China, ${ }^{1}$ Department of Obstetrics, Maternal and \\ Child Health Hospital of Weifang, 76 Young Road, Weifang 261011, People's Republic of China and ${ }^{2}$ Department of Infectious Diseases, People's Hospital of \\ Jiangbei, Nanjing, Jiangsu 210048, People's Republic of China
}

(Correspondence should be addressed to B Wang; Email: sdwfwb@yahoo.com)

\begin{abstract}
Background: Observational studies suggest possible associations between thyroid antibodies and risk of preterm delivery. However, whether thyroid antibodies are risk factors of preterm labor remains controversial. Our goal was to evaluate the associations between thyroid antibodies and risk of preterm delivery by conducting a meta-analysis of prospective cohort studies.

Methods: PubMed, Embase, and Wangfang databases were searched through January 2012 to identify studies that met pre-stated inclusion criteria. Data were extracted using standardized forms. Either a fixed- or a random-effects model was used to calculate the overall combined relative ratio (RR) with its corresponding 95\% confidence interval $(95 \%$ CI) to evaluate the relationship between thyroid antibodies and preterm delivery risk. Subgroup analyses were mainly performed by type of thyroid antibodies including thyroid peroxidase antibody (TPO-Ab) and thyroglobulin antibody (TG-Ab).

Results: Eleven prospective cohort studies involving 35467 participants were included. The combined RR of preterm delivery for pregnant women with thyroid antibodies compared with the reference group was 1.41 (95\% CI 1.08-1.84, $P=0.011)$. Subgroup analysis yielded the combined RR of preterm delivery for pregnant women with TPO-Ab compared with the reference group was $1.69(95 \%$ CI 1.19-2.41, $P=0.003)$, whereas pregnant women with positive TG-Ab had no obvious risk of preterm delivery compared with the reference group $(\mathrm{RR}=0.88,95 \%$ CI $0.60-1.29, P=0.513)$. Sensitivity analysis restricted to studies excluding women with thyroid dysfunction yielded similar results. Meta-regression analysis suggested that the status of exclusion or inclusion of women with thyroid dysfunction was the major source of heterogeneity in this meta-analysis. No evidence of publication bias was observed.

Conclusions: Current evidence suggests that the presence of TPO-Ab in pregnant women significantly increases the risk of preterm delivery.
\end{abstract}

European Journal of Endocrinology 167 455-464

\section{Introduction}

Preterm delivery is defined as birth occurring at or before 37 weeks of gestation and it remains one of the most intractable problems that contributes to perinatal morbidity and mortality in obstetric practice $(1,2)$. Despite a concerted effort to decrease the incidence of preterm delivery, there has been a relentless increased incidence over the last two decades (2). Preterm delivery results from a series of disorders, implicating maternal and fetal disease, some of which are explained and interrelated and others of which are of unknown cause $(1,2)$. Research efforts to address this problem have risen substantially over the past 10 years but have not resulted in improvements in prediction and prevention of preterm delivery (2). The incidence of preterm delivery is associated with various epidemiological and clinical risk factors including a previous preterm birth, periodontal disease, low maternal BMI, smoking, history of induced abortion, anemia, assisted reproduction, multifetal gestation, and other established risk factors, and a good understanding of risk factors may lead to the development of new therapeutic strategies $(2,3,4,5,6)$. Though there have been great advances in our understanding of the molecular and cellular pathways operative in reproductive tissues in the maintenance of uterine quiescence during pregnancy, and in initiating term and preterm labor, our knowledge of the pathophysiology of preterm delivery is limited (7). Thus, more risk factors of preterm delivery should be identified by epidemiological studies to provide good evidence for the prevention and treatment of preterm delivery $(2,3)$. 
Many epidemiological studies have investigated the links between thyroid antibodies and risk of preterm delivery $(8,9,10,11,12,13,14)$. However, the magnitudes of the association varied between studies and whether thyroid antibodies were risk factors of preterm delivery remains controversial $(8,9,10,11,12,13,14)$. Although a meta-analysis by Thangaratinam et al. (15) combined several cohort studies and reported a statistically significant relation of thyroid antibodies to preterm delivery risk, the evidence was limited because only five cohort studies were available at that time. Besides, Thangaratinam et al. (15) neither performed meta-analyses by the type of thyroid antibodies including thyroid peroxidase antibody (TPO-Ab) and thyroglobulin antibody (TG-Ab) nor explored the possible sources of obvious heterogeneity. Furthermore, whether thyroid antibodies are risk factors or merely silent markers of preterm delivery remains unclear. An improved understanding of this issue may have important public health and clinical implications given the possibility that prevention and treatment of preterm delivery might be well performed and the incidence of preterm delivery can be effectively reduced in pregnant women with thyroid antibodies. Therefore, with recently accumulating evidence, our goal was to evaluate the associations between thyroid antibodies (both TPO-Ab and TG-Ab) and risk of preterm delivery by conducting a meta-analysis of prospective cohort studies.

\section{Materials and methods}

\section{Search strategy and study selection}

We attempted to follow the proposed Meta-Analysis of Observational Studies in Epidemiology (MOOSE) guidelines to report the present meta-analysis (16). We conducted a literature search in PubMed, Embase, and Wangfang databases through January 2012 for relevant studies that tested the associations between thyroid antibodies (TPO-Ab or TG-Ab) and risk of preterm delivery. The following search terms were used: i) thyroid autoimmune antibody, thyroid autoimmune antibodies, thyroid antibody, thyroid antibodies, thyroid autoantibody, thyroid autoantibodies, thyroid globulin antibody, thyroid globulin antibodies, TPO-Ab, or thyroid peroxidase antibodies; ii) preterm delivery, preterm labor, preterm birth, premature delivery, premature labor, or premature birth; and iii) cohort study, cohort studies, prospective study, prospective studies, follow-up study, or follow-up studies. No restrictions were imposed. In addition, we reviewed the reference lists of retrieved papers and recent reviews. The reference lists of included studies were also hand searched. When necessary, additional information was sought from the authors. We first performed an initial screening of titles or abstracts. A second screening was based on full-text review.
Studies were considered eligible if they met the following criteria: i) the study design was a prospective cohort study; ii) the exposure of interest was thyroid antibodies (TPO-Ab or TG-Ab); iii) the outcome of interest was preterm delivery; and iv) relative risk (RR) and the corresponding 95\% confidence interval (95\% CI; or data to calculate them) were reported. Data from abstracts, review articles, editorials, case reports, and letters were not included. Studies performed in assisted reproductive technology (ART) pregnancies were also excluded owing to the high risk of preterm delivery and the sample size from those studies. Overlapping study or studies containing overlapping participants were excluded.

\section{Data extraction}

The key exposure variable was the presence or absence of thyroid antibodies (TPO-Ab or TG-Ab) at baseline. In all studies, women without thyroid antibodies (TPO-Ab or $\mathrm{TG}-\mathrm{Ab}$ ) served as the reference group. Outcome of interest in this study was preterm delivery. Preterm delivery was defined as birth occurring at or before 37 weeks of gestation (1). Data extraction was then performed using a standardized data collection form. We extracted any reported RR or incidence density ratios of outcomes for patients with thyroid antibodies (TPO-Ab or TG-Ab) compared with the reference group. We also extracted study characteristics for each trial and contacted primary authors to get additional information on the studies if needed. Data were recorded as follows: first author's name, year of publication, country of origin, study period, characteristics of study population and age at baseline, number of preterm delivery events and total participants, potential confounders, and ascertainments of thyroid antibodies. Two authors independently conducted the studies' selection and data extraction. Any disagreements were resolved by discussion. Information was extracted from each selected article on study characteristics, quality, and test results.

\section{Assessment of quality of methods}

We used the Newcastle-Ottawa scale to assess methodological quality of the selected studies, with the components of study design that were related to internal validity $(17,18)$. Information on adequacy of definition of cohorts, representativeness of the sample, selection and evaluation of controls, comparability, ascertainment of exposure, and outcome were evaluated for cohort studies $(17,18)$. The study was considered to have low risk of bias if it scored a maximum of four for selection, two for comparability, and three for assessment of outcome or ascertainment of exposure $(17,18)$. Any study that scored 1 or zero for selection or zero for comparability or for outcome assessment was categorized as having a high risk of bias. Studies that scored in between were rated as having a medium risk of bias. 


\section{Statistical analyses}

RR with its corresponding 95\% CI was used as a common measure of the association between thyroid antibodies (TPO-Ab or TG-Ab) and risk of preterm delivery. We calculated the overall combined RR with its $95 \%$ CI to asses the strength of the relationships between thyroid antibodies and preterm delivery risk. The significance of the combined RR was determined by the $Z$ test and a $P$ value of $<0.05$ was considered significant. In our study, two models of meta-analysis for dichotomous outcomes were conducted: the randomeffects model and the fixed-effects model $(19,20)$. The random-effects model was conducted using the DerSimonian and Laird's method, which assumed that studies were taken from populations with varying effect sizes and calculated the study weights both from in-study and between-study variances (20). The fixedeffects model was conducted using the MantelHaenszel's method, which assumed that studies were sampled from populations with the same effect size and made an adjustment to the study weights according to the in-study variance (19). To assess the between-study heterogeneity more precisely, both the $\chi^{2}$-based $Q$ statistic test (Cochran's $Q$ statistic) to test for heterogeneity and the $I^{2}$ statistic to quantify the proportion of the total variation due to heterogeneity were calculated $(21,22)$. The $I^{2}$ index expressing the percentage of the total variation across studies due to heterogeneity was calculated to assess the between-study heterogeneity. $I^{2}$ values of 25,50 , and $75 \%$ were used as evidence of low, moderate, and high heterogeneity respectively (21). If moderate or high heterogeneity existed, the randomeffects model was used to pool the results; otherwise, the fixed-effects model was used to pool the results when $I^{2}$ value was $<50 \%$. Because characteristics of participants and ascertainments of thyroid antibodies were not consistent between studies, we subsequently conducted meta-regression analysis to explore possible explanations for heterogeneity, and a $P$ value $>0.1$ was selected to indicate absence of significant heterogeneity of the estimates (23). We conducted a meta-regression analysis to explore predefined sources of heterogeneity of our primary predictor of interest. The following prespecified variables were analyzed: proportion of thyroid antibodies, multivariate adjustment (yes/no), study quality, publication year, sample size, and the status of exclusion or inclusion of women with thyroid dysfunction. Multivariate meta-regression analyses were also undertaken. Subgroup meta-analyses were performed by the type of thyroid antibodies (TPO-Ab or TG-Ab) and the status of exclusion or inclusion of women with thyroid dysfunction. We also investigated the influence of a single study on the overall risk estimate by omitting one study in each turn to validate the credibility of outcomes in this meta-analysis (24). Potential publication bias was assessed by visual inspection of the Begg's funnel plots, in which the standard error of logor of each study was plotted against its logor and an asymmetric plot suggested possible publication bias (25). In addition, we also performed the Egger linear regression test at the $P<0.10$ level of significance to assess the funnel-plot's asymmetry (26). All analyses were performed using STATA version 12.0 (StataCorp LP, College Station, TX, USA). A $P$ value $<0.05$ was considered statistically significant, except where otherwise specified.

\section{Results}

\section{Study selection and study characteristics}

We initially retrieved 33 unique citations from the PubMed, Embase, and Wangfang databases. Of these, 16 citations were excluded after the first screening based on the abstracts or titles, mainly because they were overlapping records, not cohort studies, or obviously irrelevant studies. After full-text review of the remaining 17 articles $(8,9,10,11,12,13,14,27,28$, $29,30,31,32,33,34,35,36)$, three articles were excluded because the exposure was not relevant $(32$, $33,36)$, two articles were excluded because they were not on preterm delivery $(34,35)$, and one article was excluded because of the nested case-control design (31). Finally, 11 articles involving a total of 35467 participants were included in our meta-analysis $(8,9$, $10,11,12,13,14,27,28,29$, 30). A flow chart showing the study selection was presented in Fig. 1.

The characteristics of the 11 prospective cohort studies were presented in Table 1 . These cohort studies were published between 1990 and 2011. Four studies

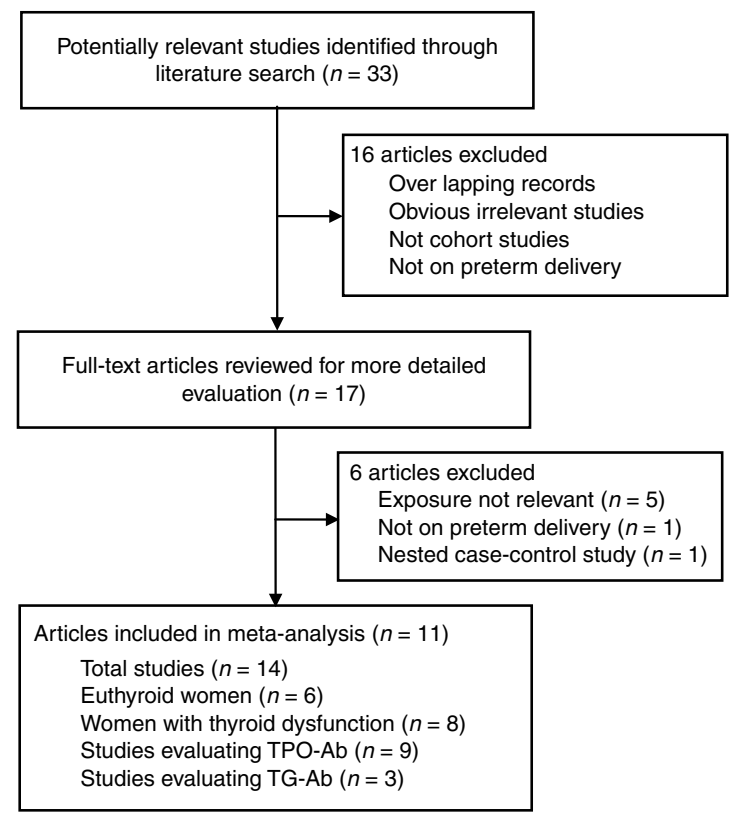

Figure 1 Flow chart of study selection. 


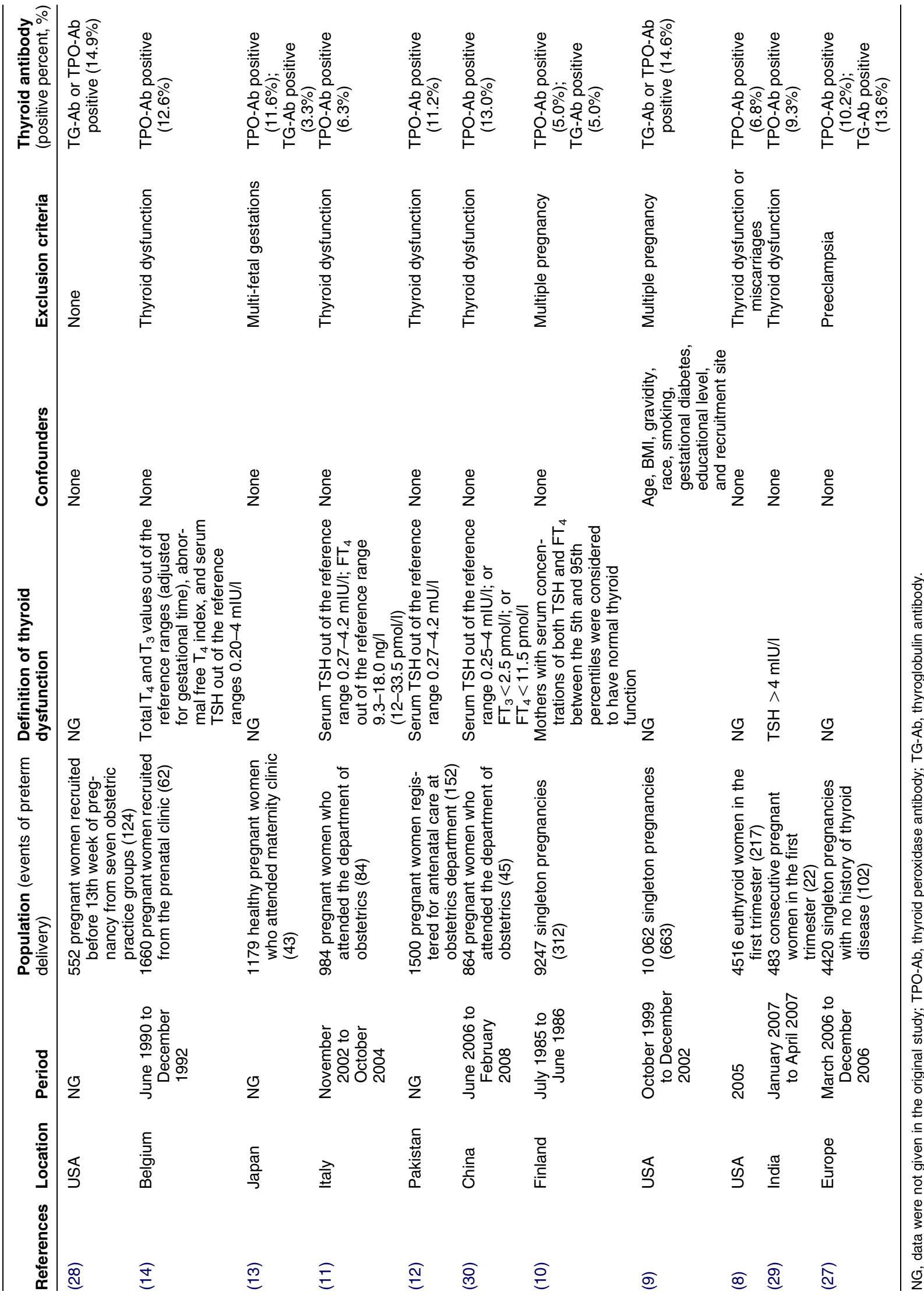


were conducted in Europe $(10,11,14,27)$, three in the United States $(8,9,28)$, and four in Asia $(12,13$, $29,30)$. The sizes of the cohorts ranged from 438 to 10062 (total 35467 ). The ascertainment of thyroid antibodies varied across studies, with most based on TPO-Ab positive. Among the 11 articles included here, nine evaluated TPO-Ab $(8,10,11,12,13,14,27,29,30)$, three evaluated TG-Ab $(10,13,27)$, and two mixed TG-Ab and TPO-Ab $(9,28)$. Three articles reported data on both TPO-Ab and TG-Ab, thus were extracted as six individual studies $(10,13,27)$. The exclusion criteria also varied across studies, with six studies excluding women with thyroid dysfunction $(8,11,12,14,29,30)$ and five studies including women with thyroid dysfunction $(9,10,13,27,28)$. The TPO-Ab positive rate ranged from 5.3 to $14.9 \%$, while the TG-Ab positive rate ranged from 3.4 to $15.8 \%$. Six studies reported information on the definition of thyroid dysfunction $(10,11,12,14,29,30)$ and only one study reported information regarding potential confounders (age, BMI, gravidity, race, smoking, gestational diabetes, educational level, and recruitment site) (9). In addition, there was an obvious difference in the definition of thyroid dysfunction in those studies included in this meta-analysis (Table 1). All 11 cohort studies had low risk of bias for selection and outcome assessment on the Newcastle-Ottawa scale (14).

\section{Synthesis analysis}

Summary of the pooled outcomes in this meta-analysis was shown in Table 2.

Thyroid antibodies and preterm delivery There were 14 individual data from 11 articles including nine on TPO-Ab, three on TG-Ab, and two on TG-Ab and TPO-Ab (8, 9, 10, 11, 12, 13, 14, 27, 28, 29, 30). Meta-analysis showed that the overall combined RR of preterm delivery risk for pregnant women with thyroid antibodies compared with the reference group was $1.41(95 \%$ CI $1.08-1.84, P=0.011$; Fig. 2). Sensitivity analyses by sequential omission of individual studies did not materially alter the overall combined RR, suggesting that the combined RR was valid and credible.

TPO-Ab and preterm delivery Nine prospective cohort studies reported relevant data on the association between TPO-Ab and preterm delivery risk $(8,10,11$, $12,13,14,27,29,30)$. Meta-analysis showed that the combined RR of preterm delivery risk for pregnant women with positive TPO-Ab compared with the reference group was 1.69 (95\% CI 1.19-2.41, $P=0.003$; Fig. 3). Sensitivity analyses by sequential omission of individual studies did not materially alter the overall combined RR, suggesting that the combined RR was valid and credible.
TG-Ab and preterm delivery Three prospective cohort studies reported relevant data on the association between TG-Ab and preterm delivery risk $(10,13,27)$. Meta-analysis showed that pregnant women with positive TG-Ab had no obvious risk of preterm delivery compared with the reference group $(\mathrm{RR}=0.88,95 \% \mathrm{CI}$ 0.60-1.29, $P=0.513$; Fig. 4). Sensitivity analyses by sequential omission of individual studies did not materially alter the overall combined RR, suggesting that the combined RR was valid and credible.

\section{Heterogeneity analysis}

There was obvious heterogeneity for the meta-analyses of both all 11 studies and the subgroup analysis of TPO-Ab $\left(I^{2}=75.0\right.$ and $71.6 \%$ respectively; Table 2$)$. Meta-regression analyses suggested that the status of exclusion or inclusion of women with thyroid dysfunction was the major source of heterogeneity in this metaanalysis (for total 11 studies, $P=0.001$; for subgroup analysis of TPO-Ab, $P=0.029$ ); however, other sources were not found by meta-regression analyses. Multivariate analyses of meta-regression were also performed but did not found further definite source of heterogeneity. For the meta-analysis of all 11 studies, the status of thyroid function could explain $63.57 \%$ of between-study variance $(P=0.001)$; for the subgroup analysis of TPO-Ab, the status of thyroid function could explain $27.05 \%$ of between-study variance $(P=0.029)$ with residual heterogeneity (residual $I^{2}=58.94 \%$ ).

As the status of exclusion or inclusion of women with thyroid dysfunction was the major source of heterogeneity, sensitivity analyses were further performed to assess the influence of the status of exclusion or inclusion of women with thyroid dysfunction on the associations between thyroid antibodies and preterm delivery risk. In studies excluding women with thyroid dysfunction, the overall combined RR of preterm delivery risk for pregnant women with thyroid antibodies or with $\mathrm{TPO}-\mathrm{Ab}$ positive status was significant (for thyroid antibodies, $\mathrm{RR}=1.98$, 95\% CI 1.29-3.04, $P=0.002$; for TPO-Ab, RR $=1.98$, 95\% CI 1.29-3.04, $P=0.002$; Table 2). In studies including women with thyroid dysfunction, none of the overall combined RRs for pregnant women with thyroid antibodies, TPO-Ab positive status, and TG-Ab positive status were statistically significant (for thyroid antibodies, $\mathrm{RR}=1.12,95 \%$ CI 0.97-1.29, $P=0.118$; for TPO-Ab, $R R=1.22$, $95 \%$ CI $0.87-1.71, P=0.243$; for TG-Ab, $\mathrm{RR}=0.88,95 \% \mathrm{CI}$ 0.60-1.29, $P=0.513$; Table 2). Subgroup analysis of women with TG-Ab positive status was not performed owing to the lack of relevant studies.

\section{Publication bias}

Visual inspection of the Begg's funnel plot did not identify substantial asymmetry (Supplementary Figure 1, see section on supplementary data given at 
Table 2 Summary of meta-analyses of thyroid antibodies and preterm delivery risk.

\begin{tabular}{|c|c|c|c|c|c|c|c|}
\hline \multirow[b]{2}{*}{ Analysis items } & \multirow[b]{2}{*}{$\begin{array}{c}\text { Studies } \\
\text { (participants) }\end{array}$} & \multicolumn{2}{|c|}{ Risk ratio } & \multirow[b]{2}{*}{ Model } & \multirow[b]{2}{*}{$I^{2}(\%)$} & \multirow[b]{2}{*}{ Absolute effect } & \multirow[b]{2}{*}{$\begin{array}{c}\text { Egger } \\
\text { test }\end{array}$} \\
\hline & & $\mathrm{RR}(95 \% \mathrm{Cl})$ & $P_{\mathrm{RR}}$ & & & & \\
\hline \multicolumn{8}{|l|}{ Thyroid antibodies } \\
\hline Total studies & $14(40609)$ & $1.41(1.08-1.84)$ & 0.011 & Random & 75.0 & $\begin{array}{l}22 \text { more per } 1000 \\
\text { (from } 4 \text { more to } \\
45 \text { more) }\end{array}$ & 0.990 \\
\hline $\begin{array}{l}\text { Studies with euthyroid } \\
\text { women }\end{array}$ & $6(7932)$ & $1.98(1.29-3.04)$ & 0.002 & Random & 71.9 & $\begin{array}{l}65 \text { more per } 1000 \\
\text { (from } 19 \text { more to } \\
136 \text { more) }\end{array}$ & 0.209 \\
\hline $\begin{array}{l}\text { Studies including } \\
\text { thyroid dysfunction }\end{array}$ & 8 (32 677) & $1.12(0.97-1.29)$ & 0.118 & Fixed & 0.0 & $\begin{array}{l}6 \text { more per } 1000 \\
\text { (from } 2 \text { fewer to } \\
15 \text { more) }\end{array}$ & 0.441 \\
\hline \multicolumn{8}{|l|}{ TPO-Ab } \\
\hline Total studies & $9(19191)$ & $1.69(1.19-2.41)$ & 0.003 & Random & 71.6 & $\begin{array}{l}22 \text { more per } 1000 \\
\text { (from } 4 \text { more to } \\
45 \text { more) }\end{array}$ & 0.134 \\
\hline $\begin{array}{l}\text { Studies with euthyroid } \\
\text { women }\end{array}$ & $6(7932)$ & $1.98(1.29-3.04)$ & 0.002 & Random & 71.9 & $\begin{array}{l}65 \text { more per } 1000 \\
\text { (from } 19 \text { more to } \\
136 \text { more) }\end{array}$ & 0.209 \\
\hline $\begin{array}{l}\text { Studies including } \\
\text { thyroid dysfunction }\end{array}$ & $3(11259)$ & $1.22(0.87-1.71)$ & 0.243 & Fixed & 0.0 & $\begin{array}{l}6 \text { more per } 1000 \\
\text { (from } 2 \text { fewer to } \\
15 \text { more) }\end{array}$ & 0.734 \\
\hline \multicolumn{8}{|l|}{$\mathrm{TG}-\mathrm{Ab}$} \\
\hline Total studies & $3(11108)$ & $0.88(0.60-1.29)$ & 0.513 & Fixed & 0.0 & $\begin{array}{l}5 \text { fewer per } 1000 \\
\text { (from } 16 \text { fewer to } \\
12 \text { more) }\end{array}$ & 0.916 \\
\hline $\begin{array}{l}\text { Studies including } \\
\text { thyroid dysfunction }\end{array}$ & $3(11$ 108) & $0.88(0.60-1.29)$ & 0.513 & Fixed & 0.0 & $\begin{array}{l}5 \text { fewer per } 1000 \\
\text { (from } 16 \text { fewer to } \\
12 \text { more) }\end{array}$ & 0.916 \\
\hline
\end{tabular}

TPO-Ab, thyroid peroxidase antibody; TG-Ab, thyroglobulin antibody; RR, risk ratio; $95 \% \mathrm{Cl}, 95 \%$ confidence interval; random, random-effects model; fixed, fixed-effects model.

the end of this article). The Egger linear regression test also showed no evidence of publication bias among studies of thyroid antibodies and risk of preterm delivery (Egger test, $P=0.990$ ). Thus, the publication bias was not evident in this meta-analysis.

\section{Discussion}

Many epidemiological studies have examined the associations between thyroid antibodies and the risk of preterm delivery, but the magnitudes of the associations vary between studies and whether thyroid antibodies are risk factors of preterm delivery remains controversial $(8,9,10,11,12,13,14)$. Although a meta-analysis combined several cohort studies and reported a statistically significant relation of thyroid antibodies to preterm delivery risk, evidence was limited because only five cohort studies were available at that time and there was unexplained heterogeneity in that meta-analysis (15). Several new studies have emerged since that metaanalysis was completed $(8,27,29,30)$, and there was a need for a new meta-analysis to comprehensively assess the associations between thyroid antibodies (TPO-Ab or $\mathrm{TG}-\mathrm{Ab}$ ) and risk of preterm delivery and to find some possible explanations for the heterogeneity in the previous meta-analysis (15). Therefore, we performed this updated meta-analysis by including 11 prospective cohort studies involving 35467 participants $(8,9,10$, $11,12,13,14,27,28,29,30)$. The combined RR of preterm delivery for pregnant women with thyroid antibodies compared with the reference group was 1.41 $(95 \%$ CI $1.08-1.84, \quad P=0.011)$. Subgroup analysis showed that the combined RR of preterm delivery for pregnant women with positive TPO-Ab compared with the reference group was 1.69 (95\% CI 1.19-2.41, $P=0.003)$, whereas pregnant women with positive TG-Ab had no obvious risk of preterm delivery compared with the reference group $(\mathrm{RR}=0.88,95 \%$ CI $0.60-1.29, P=0.513)$. There was obvious heterogeneity for the meta-analysis of both all 11 studies and the subgroup analysis of TPO-Ab $\left(I^{2}=75.0\right.$ and $71.6 \%$ respectively; Table 2). Meta-regression analyses suggested that the status of exclusion or inclusion of women with thyroid dysfunction was the major source of heterogeneity in this meta-analysis (for all 11 studies, $P=0.001$; for subgroup analysis of TPO-Ab, $P=0.029$ ). Besides, sensitivity analyses restricted to studies excluding women with thyroid dysfunction yielded similar results. Thus, current evidence suggests that pregnant women with positive TPO-Ab have higher risk of preterm delivery.

Heterogeneity is a potential problem when interpreting the results of all meta-analyses, and finding the 


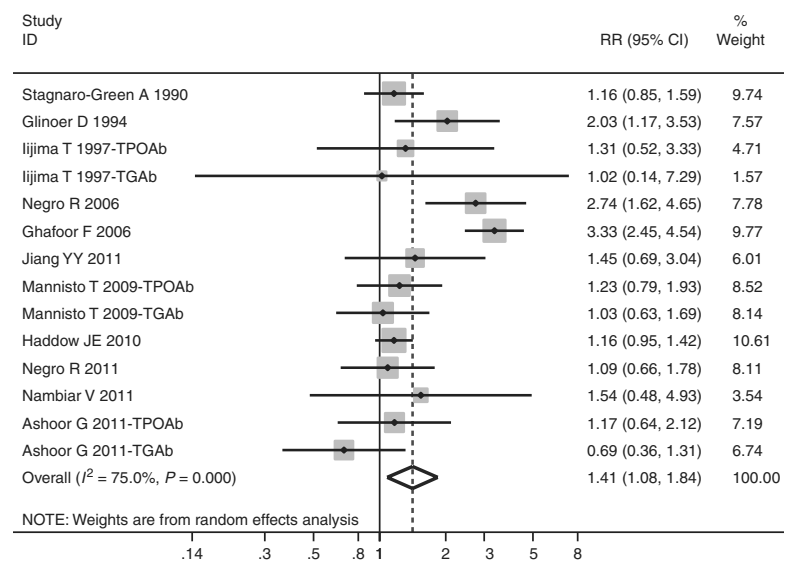

Figure 2 Forest plot showing the association between thyroid antibodies and risk of preterm delivery. $\mathrm{Cl}$, confidence interval; $\mathrm{RR}$, relative risk.

sources of heterogeneity is one of the most important goals of meta-analysis (37). There was obvious heterogeneity for the meta-analyses of both all 11 studies and the subgroup analysis of TPO-Ab (Table 2). For meta-analysis of all 11 studies, the status of thyroid function could explain $63.57 \%$ of between-study variance $(P=0.001)$; for subgroup analysis of TPO$\mathrm{Ab}$, the status of thyroid function could explain $27.05 \%$ of between-study variance $(P=0.029)$. However, the major sources of residual heterogeneity $\left(I^{2}=58.94 \%\right)$ for the subgroup analysis of TPO-Ab in euthyroid women were not found, and the heterogeneity may result from differences in the population, test assay platforms for TPO-Ab, and thresholds for test positivity and the quality features of the studies (15).

At present, the association between TPO-Ab and preterm delivery risk is not fully understood, and the underlying mechanisms involved in the association between TPO-Ab and preterm delivery risk are uncertain. It is also recognized that TPO-Ab is a marker of thyroid dysfunction, and it is also accepted that thyroid dysfunction is a risk factor of preterm delivery $(2,38)$. However, whether TPO-Ab is associated with incidence of preterm delivery remains controversial. Results from our sensitivity analysis restricted to studies excluding women with thyroid dysfunction show that euthyroid women with TPO-Ab positive status have higher risk of preterm delivery compared with the reference group $(\mathrm{RR}=1.98,95 \%$ CI 1.29-3.04, $P=0.002$ ), and this suggests that TPO-Ab is probably a risk factor for preterm delivery. Pregnancy is an inflammatory process involving a shift in the regulation of cytokine networks within the local placental-decidual environment $(4,39)$. Dysregulation of local inflammatory processes can be associated with premature delivery. The frequent presence of TPO-Ab in autoimmune diseases supports a global immune dysfunction being relevant to TPO-Ab $(40,41,42)$. The presence of TPO-Ab can reflect a dysregulated activity of the immune system at the fetal-maternal interface and might be a marker of immune dysfunction (43). Thus, plausible biological explanations exist to support the association between TPO-Ab and preterm delivery risk.

A major strength of our study is that all the included original studies use a prospective cohort design, which eliminates the possibility of reverse causation and minimizes selection bias. Moreover, the association of TPO-Ab with risk of preterm delivery persists and remains statistically significant in sensitivity analyses restricted to studies excluding women with thyroid dysfunction. In addition, with the accumulating evidence, enlarged sample size, and elimination of bias caused by thyroid function, we have enhanced statistical power to provide more precise and reliable risk estimates. The prevalence of TPO-Ab ranged from 5.26 to $14.89 \%$, and the overall combined RR of preterm delivery risk for euthyroid pregnant women with TPO-Ab compared with the reference group was 1.98 (95\% CI 1.29-3.04, $P=0.002)$. This improved understanding of the association between TPO-Ab and preterm delivery risk may have important public health and clinical implications given the possibility that prevention of preterm delivery in pregnant women with TPO-Ab might be effectively achieved (1). Negro et al. reported that euthyroid pregnant women who are positive for TPOAb have an increased risk of miscarriage and premature deliveries, and substitutive treatment with $\mathrm{L}-\mathrm{T}_{4}$ is able to lower the chance of miscarriage and premature delivery in women with TPOAb positive status (11). Thus, for women with TPOAb positive status, the chance of premature delivery could be lowered by preventive therapy. Thus, TPO-Ab could be a risk factor of preterm delivery and TPO-Ab testing may be a good marker for the prevention of preterm delivery $(2,3)$.

Several limitations should be taken into account in the interpretation of results from this meta-analysis. First, there was obvious difference in the test assay platforms for TPO-Ab and thresholds for test positivity used between studies, which could result in the

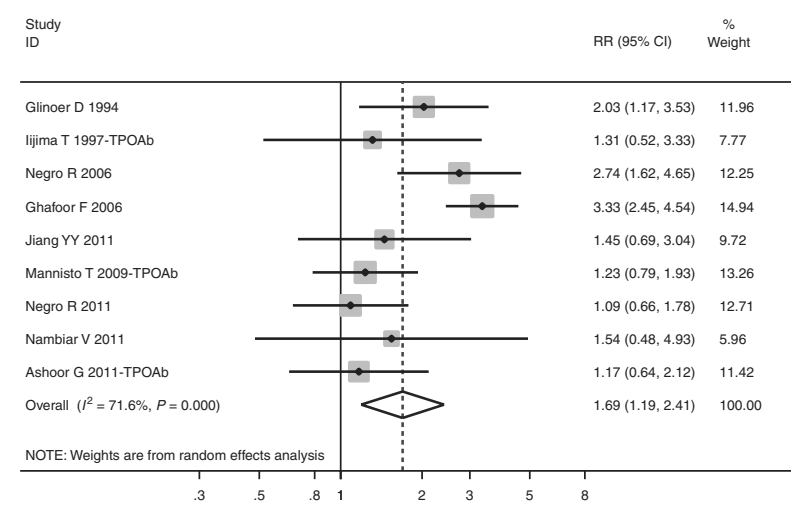

Figure 3 Forest plot showing the association between TPO-Ab and risk of preterm delivery. $\mathrm{Cl}$, confidence interval; $\mathrm{RR}$, relative risk. 


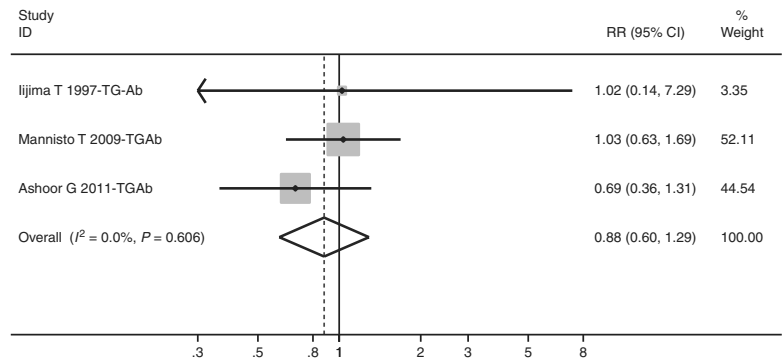

Figure 4 Forest plot showing no association between TG-Ab positive status and risk of preterm delivery. $\mathrm{Cl}$, confidence interval; $\mathrm{RR}$, relative risk.

heterogeneity in this meta-analysis. Besides, definition of thyroid dysfunction in pregnancy has evolved over the years and there was obvious difference in the definition of thyroid dysfunction in those studies included in the present meta-analysis. The differences in both the definition of thyroid dysfunction in pregnancy and the threshold for test positivity of TPO$\mathrm{Ab}$ or TG-Ab may cause the heterogeneity and should be taken into account in the interpretation of results. Further studies may identify the more reasonable assay platform or threshold for test positivity to better predict the risk of preterm delivery. Secondly, our meta-analysis used unadjusted RRs from individual cohorts owing to the lack of adjusted RRs. It is no doubt RRs adjusted by potential confounders (smoking, age, history of induced abortion, history of preterm delivery, multifetal gestation, and other established risk factors) are suitable to get a more precise estimate. Further studies can investigate the effects of thyroid antibodies on preterm delivery risk by adjusting the potential confounders. Besides, the differences in the potential confounders prove to be helpful in the heterogeneity exploration, but owing to the limited reported information regarding potential confounders in the included studies, we were unable to identify whether those differences were a source of heterogeneity in this meta-analysis. Thirdly, there was a lack of data on the association between $\mathrm{TG}-\mathrm{Ab}$ and preterm delivery risk in euthyroid women. Though subgroup meta-analysis of three studies suggested that TG-Ab positive pregnant women had no obvious risk of preterm delivery compared with the reference group $(\mathrm{RR}=0.88,95 \%$ CI $0.60-1.29$, $P=0.513)$, those three did not exclude women with thyroid dysfunction and the accurate estimate might be biased by thyroid dysfunction $(10,13,27)$. Besides, this estimate might be an over-interpretation of findings, given that the analysis is based on the results of the quantitative synthesis of only three studies and probably underpowered. Thus, further studies are needed to assess the possible association between $\mathrm{TG}-\mathrm{Ab}$ and preterm delivery risk in euthyroid women. Fourthly, there is a broad range of 'TSH normality' and obvious difference in the TSH levels in women with $\mathrm{TPO}-\mathrm{Ab}$ and women without TPO-Ab even in studies excluding women with thyroid dysfunction. Among those six studies excluding women with thyroid dysfunction $(8,11,12,14,29,30)$, only Negro et al. compared the TSH levels in women with TPO-Ab with those without TPO-Ab and reported higher TSH levels in women with positive TPO-Ab $(1.7 \pm 0.5 \mathrm{mIU} / \mathrm{l})$ compared with women without TPO-Ab (1.1 \pm $0.4 \mathrm{mIU} / \mathrm{l} ; P<0.05)(11)$, and the difference of TSH levels might bias the real estimate. However, none of those studies reported RRs adjusted by TSH levels, and further studies are needed to assess RRs adjusted by TSH levels, which may further identify whether TPO-Abs is a risk factor independent of conventional risk factors of preterm delivery. Finally, the associations between other types of antibodies and preterm delivery risk are interesting and are worth investigating, such as antinuclear antibody, anti-DNA antibody, and antimitochondrial antibody (13). However, there is little literature assessing the association between other types of antibodies and preterm delivery risk, and we are unable to perform a meta-analysis owing to the limited studies. More studies are needed to assess the possible associations between other types of antibodies and preterm delivery risk in the future. Besides, there is no literature published to assess the association between TPO-Abs and preterm delivery risk in ART pregnancies up to now. Further studies are needed to assess the possible association in ART pregnancies.

This meta-analysis of prospective cohort studies suggests that TPO-Ab in pregnant women significantly increases the risk of preterm delivery. Further studies can investigate the effects of other thyroid antibodies on preterm delivery risk by adjusting the potential confounders.

\section{Supplementary data}

This is linked to the online version of the paper at http://dx.doi.org/10. 1530/EJE-12-0379.

\section{Declaration of interest}

The authors declare that there is no conflict of interest that could be perceived as prejudicing the impartiality of the research reported.

\section{Funding}

This research did not receive any specific grant from any funding agency in the public, commercial or not-for-profit sector.

\section{Acknowledgements}

The authors thank Mr Beicheng Sun in Nanjing Medical University for his kind help with statistics.

\section{References}

1 Simhan HN \& Caritis SN. Prevention of preterm delivery. New England Journal of Medicine 2007357 477-487. (doi:10.1056/ NEJMra050435) 
2 Goldenberg RL, Culhane JF, Iams JD \& Romero R. Epidemiology and causes of preterm birth. Lancet 2008 371 75-84. (doi:10.1016/S0140-6736(08)60074-4)

3 Iams JD, Romero R, Culhane JF \& Goldenberg RL. Primary, secondary, and tertiary interventions to reduce the morbidity and mortality of preterm birth. Lancet $2008 \quad 371$ 164-175. (doi:10.1016/S0140-6736(08)60108-7)

4 Kyrklund-Blomberg NB \& Cnattingius S. Preterm birth and maternal smoking: risks related to gestational age and onset of delivery. American Journal of Obstetrics and Gynecology 1998179 1051-1055. (doi:10.1016/S0002-9378(98)70214-5)

5 Bloom SL, Yost NP, McIntire DD \& Leveno KJ. Recurrence of preterm birth in singleton and twin pregnancies. Obstetrics and Gynecology 200198 379-385. (doi:10.1016/S0029-7844 (01)01466-1)

6 Scanlon KS, Yip R, Schieve LA \& Cogswell ME. High and low hemoglobin levels during pregnancy: differential risks for preterm birth and small for gestational age. Obstetrics and Gynecology 2000 96 741-748. (doi:10.1016/S0029-7844(00)00982-0)

7 Smith R. Parturition. New England Journal of Medicine 2007356 271-283. (doi:10.1056/NEJMra061360)

8 Negro R, Schwartz A, Gismondi R, Tinelli A, Mangieri T \& Stagnaro-Green A. Thyroid antibody positivity in the first trimester of pregnancy is associated with negative pregnancy outcomes. Journal of Clinical Endocrinology and Metabolism 201196 E920-E924. (doi:10.1210/jc.2011-0026)

9 Haddow JE, Cleary-Goldman J, McClain MR, Palomaki GE, Neveux LM, Lambert-Messerlian G, Canick JA, Malone FD, Porter TF, Nyberg DA, Bernstein PS \& D'Alton ME. Thyroperoxidase and thyroglobulin antibodies in early pregnancy and preterm delivery. Obstetrics and Gynecology 2010116 58-62. (doi:10.1097/AOG.0b013e3181e10b30)

10 Mannisto T, Vaarasmaki M, Pouta A, Hartikainen AL, Ruokonen A, Surcel HM, Bloigu A, Jarvelin MR \& SuvantoLuukkonen E. Perinatal outcome of children born to mothers with thyroid dysfunction or antibodies: a prospective population-based cohort study. Journal of Clinical Endocrinology and Metabolism 2009 94 772-779. (doi:10.1210/jc.2008-1520)

11 Negro R, Formoso G, Mangieri T, Pezzarossa A, Dazzi D \& Hassan H. Levothyroxine treatment in euthyroid pregnant women with autoimmune thyroid disease: effects on obstetrical complications. Journal of Clinical Endocrinology and Metabolism 200691 2587-2591. (doi:10.1210/jc.2005-1603)

12 Ghafoor F, Mansoor M, Malik T, Malik MS, Khan AU, Edwards R \& Akhtar W. Role of thyroid peroxidase antibodies in the outcome of pregnancy. Journal of College of Physicians and Surgeons Pakistan $200616468-471$.

13 Iijima T, Tada H, Hidaka Y, Mitsuda N, Murata Y \& Amino N. Effects of autoantibodies on the course of pregnancy and fetal growth. Obstetrics and Gynecology $1997 \quad 90$ 364-369. (doi:10.1016/S0029-7844(97)00283-4)

14 Glinoer D, Riahi M, Grun JP \& Kinthaert J. Risk of subclinical hypothyroidism in pregnant women with asymptomatic autoimmune thyroid disorders. Journal of Clinical Endocrinology and Metabolism 199479 197-204. (doi:10.1210/jc.79.1.197)

15 Thangaratinam S, Tan A, Knox E, Kilby MD, Franklyn J \& Coomarasamy A. Association between thyroid autoantibodies and miscarriage and preterm birth: meta-analysis of evidence. BMJ 2011342 d2616. (doi:10.1136/bmj.d2616)

16 Stroup DF, Berlin JA, Morton SC, Olkin I, Williamson GD, Rennie D, Moher D, Becker BJ, Sipe TA \& Thacker SB. Meta-analysis of observational studies in epidemiology: a proposal for reporting. Meta-analysis Of Observational Studies in Epidemiology (MOOSE) group. Journal of the American Medical Association 2000283 2008-2012. (doi:10.1001/jama.283.15.2008)

17 Wells G, Shea B, O'connell D, Peterson J, Welch V, Losos M \& Tugwell P. The Newcastle-Ottawa Scale (NOS) for assessing the quality of nonrandomised studies in meta-analyses. Proc 3rd Symposium on Systematic Reviews, Oxford, UK, 2000.
18 Asbridge M, Hayden JA \& Cartwright JL. Acute cannabis consumption and motor vehicle collision risk: systematic review of observational studies and meta-analysis. BMJ 2012344 e 536. (doi:10.1136/bmj.e536)

19 Mantel N \& Haenszel W. Statistical aspects of the analysis of data from retrospective studies of disease. Journal of the National Cancer Institute 195922 719-748.

20 DerSimonian R \& Laird N. Meta-analysis in clinical trials. Controlled Clinical Trials 19867 177-188. (doi:10.1016/01972456(86)90046-2)

21 Higgins JP, Thompson SG, Deeks JJ \& Altman DG. Measuring inconsistency in meta-analyses. BMJ $2003 \quad 327$ 557-560. (doi:10.1136/bmj.327.7414.557)

22 Cochran WG. The combination of estimates from different experiments. Biometrics 195410 101-129. (doi:10.2307/ 3001666)

23 Thompson SG \& Higgins J. How should meta-regression analyses be undertaken and interpreted? Statistics in Medicine 200221 1559-1573. (doi:10.1002/sim.1187)

24 Tobias A. Assessing the influence of a single study in the metaanalysis estimate. Stata Technical Bulletin 1999 8 15-17.

25 Stuck AE, Rubenstein LZ \& Wieland D. Bias in meta-analysis detected by a simple, graphical test, Asymmetry detected in funnel plot was probably due to true heterogeneity. BMJ 1998316469. (doi:10.1136/bmj.316.7129.469)

26 Egger M, Davey Smith G, Schneider M \& Minder C. Bias in meta-analysis detected by a simple, graphical test. BMJ 1997315 629-634. (doi:10.1136/bmj.315.7109.629)

27 Ashoor G, Maiz N, Rotas M, Jawdat F \& Nicolaides KH. Maternal thyroid function at 11-13 weeks of gestation and spontaneous preterm delivery. Obstetrics and Gynecology 2011117 293-298. (doi:10.1097/AOG.0b013e318205152c)

28 Stagnaro-Green A, Roman SH, Cobin RH, el-Harazy E, Alvarez-Marfany M \& Davies TF. Detection of at-risk pregnancy by means of highly sensitive assays for thyroid autoantibodies. Journal of the American Medical Association 1990264 1422-1425. (doi:10.1001/jama.1990.03450110068029)

29 Nambiar V, Jagtap VS, Sarathi V, Lila AR, Kamalanathan S, Bandgar TR, Menon PS \& Shah NS. Prevalence and impact of thyroid disorders on maternal outcome in Asian-Indian pregnant women. Journal of Thyroid Research 20112011429097. (doi:10.4061/2011/429097)

30 Jiang YY, Wu YJ, Xu YH, Luo Y, Xu XM, Shen YH, Dong YY \& Zhao JH. Investigation onto thyroid dysfunction autoantibody screening in second third trimesters of pregnancy. Chinese Journal of Endocrinology and Metabolism 201127 816-820.

31 Stagnaro-Green A, Chen X, Bogden JD, Davies TF \& Scholl TO. The thyroid and pregnancy: a novel risk factor for very preterm delivery. Thyroid 200515 351-357. (doi:10.1089/thy. 2005.15.351)

32 Radetti G, Renzullo L, Gottardi E, D’Addato G \& Messner H. Altered thyroid and adrenal function in children born at term and preterm, small for gestational age. Journal of Clinical Endocrinology and Metabolism 200489 6320-6324. (doi:10.1210/jc.2003032185)

33 Negro R, Schwartz A, Gismondi R, Tinelli A, Mangieri T \& Stagnaro-Green A. Increased pregnancy loss rate in thyroid antibody negative women with TSH levels between 2.5 and 5.0 in the first trimester of pregnancy. Journal of Clinical Endocrinology and Metabolism 201095 E44-E48. (doi:10.1210/jc.2010-0340)

34 Jiskra J, Bartakova J, Holinka S, Limanova Z, Springer D, Fait T, Antosova M, Telicka Z \& Potlukova E. Low concordance between positive antibodies to thyroperoxidase and thyroid ultrasound autoimmune pattern in pregnant women. Endocrine Journal 2011 58 849-859. (doi:10.1507/endocrj.EJ11-0026)

35 Bizzaro N, Antico A, Musso M, Platzgummer S, Camogliano L, Tozzoli R \& Villalta D. A prospective study of 1038 pregnancies on the predictive value of anti-annexin $\mathrm{V}$ antibodies for fetal loss. Annals of the New York Academy of Sciences 20051050 348-356. (doi:10.1196/annals.1313.037) 
36 Kumar A, Agarwal K, Gupta RK \& Kar P. Obstetric outcome in women with hepatitis $\mathrm{C}$ virus infection and thyroid dysfunction. Acta Obstetricia et Gynecologica Scandinavica 200988 1133-1137. (doi:10.1080/00016340903144220)

37 Ioannidis JP, Patsopoulos NA \& Evangelou E. Uncertainty in heterogeneity estimates in meta-analyses. BMJ $2007 \mathbf{3 3 5}$ 914-916. (doi:10.1136/bmj.39343.408449.80)

38 van den Boogaard E, Vissenberg R, Land JA, van Wely M, van der Post JA, Goddijn M \& Bisschop PH. Significance of (sub)clinical thyroid dysfunction and thyroid autoimmunity before conception and in early pregnancy: a systematic review. Human Reproduction Update 201117 605-619. (doi:10.1093/humupd/dmrO24)

39 Weetman AP. Immunity, thyroid function and pregnancy: molecular mechanisms. Nature Reviews Endocrinology 20106 311-318. (doi:10.1038/nrendo.2010.46)

40 Nakamura H, Usa T, Motomura M, Ichikawa T, Nakao K, Kawasaki E, Tanaka M, Ishikawa K \& Eguchi K. Prevalence of interrelated autoantibodies in thyroid diseases and autoimmune disorders. Journal of Endocrinological Investigation $2008 \mathbf{3 1}$ 861-865.

41 Gleicher N, Barad D \& Weghofer A. Functional autoantibodies, a new paradigm in autoimmunity? Autoimmunity Reviews 20077 42-45. (doi:10.1016/j.autrev.2007.06.001)

42 Scofield RH. Autoantibodies as predictors of disease. Lancet 2004 363 1544-1546. (doi:10.1016/S0140-6736(04)16154-0)

43 Alexander EK. Autoimmunity: thyroid autoantibodies and pregnancy risk. Nature Reviews Endocrinology 20117 501-502. (doi:10.1038/nrendo.2011.116)

Received 3 May 2012

Revised version received 16 July 2012

Accepted 23 July 2012 